\title{
note
}

\section{Contamination atmosphérique par les produits de radiolyse des solutions aqueuses de méthionine ${ }^{35} \mathbf{S}$}

\author{
F. SIMONNET et G. SIMONNET *
}

\begin{abstract}
RÉSUMÉ A l'ouverture d'un flacon de méthionine ${ }^{35} \mathrm{~S}$ il peut s'échapper de 35 à $60 \mathrm{kBq}$ de radioactivité sous forme volatile selon le degré de radiolyse. Bien que cela ne représente que le $1 / 500^{e}$ de la LAl (limite d'incorporation) il est préférable de manipuler sous hotte ventilée et si possible de prélever sans ouvrir le flacon.
\end{abstract}

ABSTRACT On the opening of a container of ${ }^{35} \mathrm{~S}$ methionine, 35 to $60 \mathrm{kBq}$ of radioactivity; depending on the extent of radiolysis, can escape in the form of volatle compounds. Although this represents only $0.2 \%$ of the ALI (annual limit on intake) it is preferable to work with a ventilated fume hood and, if possible, to use a syringe to sample the labelled methionine.

\section{INTRODUCTION}

MEISENHELDER et HUNTER (1988) [3] puis SMITH et coll. (1989) [8] ont attiré l'attention sur le fait que l'ouverture d'un flacon de méthionine ${ }^{35} S$ ou de $\alpha$-thio dATP ${ }^{35} S$ présentait pour l'opérateur des risques de contamination interne par inhalation. Ces substances n'étant pas ellesmêmes volatiles, ce sont les produits de dégradation $[3,6]$ formés par radiolyse qui provoquent une contamination atmosphérique. Interrogés sur ces problèmes par nos collègues biochimistes, nous avons cherché à évaluer ces risques dans le cas de la méthionine ${ }^{35} \mathrm{~S}$ et ceci en fonction du temps et des conditions de stockage du produit marqué.

* INSTN, CEN Saclay, 91191 Gif-sur-Yvette Cedex (France). 


\section{MATÉRIEL ET MÉTHODES}

La $L^{35} S$ méthionine provenant de chez Amersham est en solution aqueuse d'acétate de potassium $20 \mathrm{mM}$ contenant $0,1 \%$ de 2-mercaptoéthanol; sa radioactivité spécifique est de 30 ou $53 \mathrm{TBq} / \mathrm{millimole.} \mathrm{La}$ $L_{-}^{35} S$ méthionine provenant de chez NEN-Dupont de Nemours et du CEASaclay (France) est en tampon tricine de $50 \mathrm{mM}$. La radioactivité spécifique est respectivement de 42,6 et $44,3 \mathrm{TBq} / \mathrm{millimole}$. Tous ces produits sont livrés congelés et sous atmosphère d'azote.

Certains lots de $L^{35} S$ méthionine ont été fractionnés. Chaque fraction n'est alors décongelée qu'une fois. D'autres lots ont subi des étapes de congélation - décongélation successives. L'atmosphère d'azote n'a pas été rétablie afin de créer une situation défavorable quant à la radiolyse. La conservation a été faite à $-80^{\circ} \mathrm{C}$ pendant 20 semaines ou à $-20^{\circ} \mathrm{C}$ pendant 6 semaines.

\section{EXPÉRIMENTATION}

La mesure de la quantité de radioactivité volatile présente dans un flacon de méthionine ${ }^{35} \mathrm{~S}$ est réalisée au moyen du dispositif suivant : le bouchon de caoutchouc ou de bakélite du flacon est percé de deux orifices ; l'un permet une entrée d'air, l'autre l'aspiration du contenu gazeux du flacon. La circulation d'air est obtenue au moyen d'une pompe péristaltique ; le débit est de 1,6 ml par minute. La mesure de radioactivité du gaz circulant se fait par scintillation liquide en continu (appareil Radiomatic - Flotec, La Queue lez-Yvelines, 78, France). La cellule de mesure a un volume de $2,5 \mathrm{ml}$. Le débit de liquide scintillant est de $3 \mathrm{ml}$ par minute. Les liquides scintillants utilisés sont Ready-Organic de Beckman et Rotiszint 1100 de Roth. Après la détection en continu, le liquide scintillant est collecté et $10 \mathrm{ml}$ sont prélevés ; la radioactivité est alors mesurée avec un compteur à scintillation liquide Intertechnique SL 4000 (Kontron, Montigny-le-Bretonneux, 78, France).

Pour chaque échantillon, l'efficacité de comptage est déterminée par rapport à des courbes de correction d'affaiblissement lumineux (quenching) établies précédemment sur ce compteur à l'aide d'un standard $\mathrm{H}_{2}$ ${ }^{35} \mathrm{SO}_{4}(7)$. Le standard a une activité volumique de $9,3210^{4} \mathrm{~Bq} / \mathrm{ml}$ et provient de New england nuclear (Boston, MA).

Au cours de l'opération de balayage de gaz, une partie très importante de la radioactivité volatile se fixe sur les parois des tubes en plastique siliconé de la pompe péristaltique et sur les parois des tubes en téflon du compteur (ces phénomènes d'accrochage du ${ }^{35} \mathrm{~S}$ sur les matières plastiques sont extrêmement fréquents). Les produits radioactifs fixés sur les parois de tube sont élués par un mélange éthanol-méthanol dont la radioactivité est ensuite mesurée.

La radioactivité volatile dans le flacon de méthionine ${ }^{35} \mathrm{~S}$ est la somme des radioactivités mesurées en sortie de comptage en continu plus celle éluée à partir des tubes de transfert. 


\section{RÉSULTATS ET DISCUSSION}

Une mesure de la radioactivité volatile présente dans les flacons de méthionine ${ }^{35} \mathrm{~S}$ est effectuée dès réception du produit, avant stockage. La quantité de radioactivité volatile est alors de 35 à $45 \mathrm{kBq}$ (tableau 1), quelles que soient l'origine et la quantité totale de radioactivité dans le flacon : 37 à $185 \mathrm{MBq}$. Le fait que la quantité de radioactivité volatile ne soit pas proportionnelle à la quantité de radioactivité totale paraît surprenant. Cependant, si la radioactivité volumique est du même ordre de grandeur, d'autres paramètres comme la forme du flacon, la température ambiante au moment du balayage d'air à l'intérieur du flacon, ne sont pas identiques $\left(19\right.$ à $\left.25^{\circ} \mathrm{C}\right)$. Le même ordre de grandeur de $37 \mathrm{kBq}$ a été observé par MEISENHELDER et HUNTER [3] pour une quantité de méthionine ${ }^{35} \mathrm{~S}$ de $296 \mathrm{MBq}$.

TABLEAU ।

Radioactivité volatile dégagée par diffẻrents flacons de méthionine ${ }^{35} \mathbf{S}$. Les quantités de méthionine ${ }^{35} \mathrm{~s}$, les conditions de stockage température et temps de conservation sont différentes

\begin{tabular}{|c|c|c|c|c|c|c|}
\hline $\begin{array}{c}\text { Quantité } \\
\text { Méthionine } \\
\text { en MBq }\end{array}$ & $\begin{array}{c}\text { Temps } \\
\text { stockage } \\
\text { semaines }\end{array}$ & $\begin{array}{c}\text { Température } \\
\text { de } \\
\text { conservation } \\
{ }^{\circ} \mathrm{C}\end{array}$ & $\begin{array}{c}\text { Décongélation } \\
\text { répétée }\end{array}$ & $\begin{array}{c}\text { Activité } \\
\text { volumique } \\
\mathrm{Bq} / \mathrm{ml}\end{array}$ & $\begin{array}{c}\text { Activité } \\
\text { spécifique } \\
\text { TBq/mmole }\end{array}$ & $\begin{array}{c}\text { Radioactivité } \\
\text { volatile } \\
\mathrm{kBq}\end{array}$ \\
\hline 185 & 0 & - & - & 555 & 53,4 & $35,8 \pm 5$ \\
37 & 0 & - & - & 560 & 29,6 & $33,0 \pm 5$ \\
37 & 0 & - & - & 415 & 44,3 & $45 \pm 5$ \\
133 & 6 & -20 & + & 403 & 38 & $63 \pm 6$ \\
30 & 6 & -80 & - & 300 & 31,8 & $42 \pm 4$ \\
13 & 18 & -80 & - & 130 & 15,4 & $46 \pm 5$ \\
\hline
\end{tabular}

Lorsque la méthionine ${ }^{35} \mathrm{~S}$ est conservée à $-20^{\circ} \mathrm{C}$ en présence d'air et qu'une décongélation est effectuée chaque semaine, la quantité de radioactivité volatile recueillie à chaque décongélation est du même ordre de grandeur les trois premières semaines : 35 à $45 \mathrm{kBq}$, puis augmente jusqu'à $63 \pm 6 \mathrm{kBq}$ la $6^{\text {ème }}$ semaine. Si la conservation est effectuée à $-80^{\circ} \mathrm{C}$ et sans décongélation répétée de l'échantillon, la quantité de radioactivité volatile est moindre : $46 \mathrm{kBq} \pm 5$ après 18 semaines. Ces résultats peuvent être rapprochés de ceux de SHIMAZU et al [6]. Ces auteurs ont étudié les dommages subis par la méthionine lorsqu'elle est irradiée et ont observé que la production de substances volatiles telles que $\mathrm{CH}_{3} \mathrm{SH}$, et $\mathrm{H}_{2} \mathrm{~S}$ augmentait en fonction du débit de dose reçue, donc de la radiolyse. Les fabricants de produits recommandent pour limiter la radiolyse le stockage à $-80^{\circ} \mathrm{C}$ et le fractionnement des produits pour éviter les décongélations successives. Ces recommandations sont donc à respecter impérativement.

II n'a pas été mis en évidence de différence entre méthionine ${ }^{35} \mathrm{~S}$ en solution aqueuse d'acétate de $\mathrm{K}$ ou en tampon tricine. 
A partir des résultats de mesure des quantités de radioactivité volatile effectuées dans les différentes conditions, on peut évaluer les risques d'exposition interne pour un travailleur. La méthode de mesure que nous avons utilisée a pour conséquence de surévaluer le risque. En effet, le balayage gazeux du contenu du flacon fait ressortir de celui-ci une quantité de produits volatils radioactifs certainement supérieure à celle qui s'échappe par simple ouverture du flacon. Une contamination atmosphérique qui provoque une inhalation de $60 \mathrm{kBq}$ entraîne une exposition interne inférieure à $1 / 1000$ de la limite annuelle d'incorporation (LAI). Dans ce calcul on a pris comme valeur de la $\mathrm{LAl} d \mathrm{du}^{35} \mathrm{~S}: 8.10^{7} \mathrm{~Bq}(2)$. MINSKI M. et VENNART J. [4], puis PIECHOWSKI J. et MENNOUX B. [5] préconisent pour la méthionine ${ }^{35} \mathrm{~S}$ une valeur de $\mathrm{LAl}$ de $4.10^{7} \mathrm{~Bq}$. Si l'on prend cette valeur, une contamination de $60 \mathrm{kBq}$ représente alors une exposition interne inférieure à $1 / 500$ de la LAl. II a été montré que l'organe cible est la moelle osseuse [4].

Bien que le niveau de contamination soit relativement faible, il est néanmoins souhaitable de suivre les recommandations formulées par MEISENHELDER et HUNTER [3] ; elles consistent à manipuler sous hotte ventilée et, si cela est possible, à ne pas ouvrir le flacon pour effectuer le prélèvement ; celui-ci sera fait à l'aide d'une seringue. Ces deux précautions permettent de supprimer tous les risques de contamination atmosphérique. II est aussi possible d'utiliser de la méthionine ${ }^{35} \mathrm{~S}$ additionnée d'acide pyridine-3-4 dicarboxylique ; ce composé a pour but de réduire la vitesse de radiolyse et ainsi de diminuer la quantité de produits radioactifs volatils. La présence de ce stabilisant ne modifie pas l'incorporation de méthionine ${ }^{35} \mathrm{~S}$ dans un système de traduction de réticulocytes de lapin ou de germes de blé [1]. Néanmoins, pour d'autres systèmes d'incorporation, il peut être nécessaire de vérifier que le stabilisant ne modifie pas la réaction.

\section{REMERCIEMENTS}

Les auteurs remercient l'Institut Pasteur (Paris - France) et le service des molécules marquées du CEN-Saclay qui ont fourni gracieusement la méthionine ${ }^{35} \mathrm{~S}$.

\section{RÉFÉRENCES}

[1] Amersham Research News. - 1989, vol. 2 issue 1, 8.

[2] Journal Officiel. - 1986 (2 octobre). Décret $n^{\circ} 86-1103$ relatif à la protection des travailleurs contre les dangers des rayonnements ionisants.

[3] MEISENHELDER J. and HUNTER T. - Radioactive protein - labelling techniques. Nature, 1988. 335, 120.

[4] MINSKI M. and VENNART J. - Maximum permissible intakes ${ }^{35} \mathrm{~S}$ méthionine and ${ }^{35} \mathrm{~S}$ sodium sulphate deduced from experiments on rats. Health Physics, $1971,20,1-9$. 
CONTAMINATION PAR LES PRODUITS DE RADIOLYSE DES SOLUTIONS AQUEUSES DE MÉTHIONINE ${ }^{35} \mathrm{~S}$

[5] PIECHOWSKI J. and MENNOUX B. - Surveillance par mesure de l'excrétion urinaire après exposition au ${ }^{14} \mathrm{C},{ }^{32} \mathrm{P},{ }^{35} \mathrm{~S},{ }^{45} \mathrm{Ca},{ }^{125} \mathrm{I}$. Rapport CEA DPS/LEB 87/27 1987.

[6] SHIMAZU F., KUMTA U.S. TAPPEL A.L. - Radiation damage to methionine and its derivatives. Rad. Res. 1964, 22, 276-287.

[7] SIMONNET F. and BOCQUET C. - Radiotoxicological detection of ${ }^{35} \mathrm{~S}$. Health Physics, 1986, 50, 140-143.

[8] SMITH I., FURST V. and HOLTON J. - Hasards of sulphur. Nature, 1989, 337, 696. 